\title{
Exchange Bias in Magnetic Topological Insulator Superlattices
}

\author{
Jieyi Liu,* Angadjit Singh, Yu Yang Fredrik Liu, Adrian Ionescu, Balati Kuerbanjiang, \\ Crispin H. W. Barnes, and Thorsten Hesjedal*
}

Cite This: Nano Lett. 2020, 20, 5315-5322

Read Online

ACCESS | W Metrics \& More | 国 Article Recommendations | st Supporting Information

ABSTRACT: Magnetic doping and proximity coupling can open a band gap in a topological insulator (TI) and give rise to dissipationless quantum conduction phenomena. Here, by combining these two approaches, we demonstrate a novel TI superlattice structure that is alternately doped with transition and rare earth elements. An unexpected exchange bias effect is unambiguously confirmed in the superlattice with a large exchange bias field using magneto-transport and magneto-optical techniques. Further, the Curie temperature of the Cr-doped layers in the superlattice is found to increase by $60 \mathrm{~K}$ compared to a Cr-doped single-layer film. This result is supported by density-functionaltheory calculations, which indicate the presence of antiferromag-

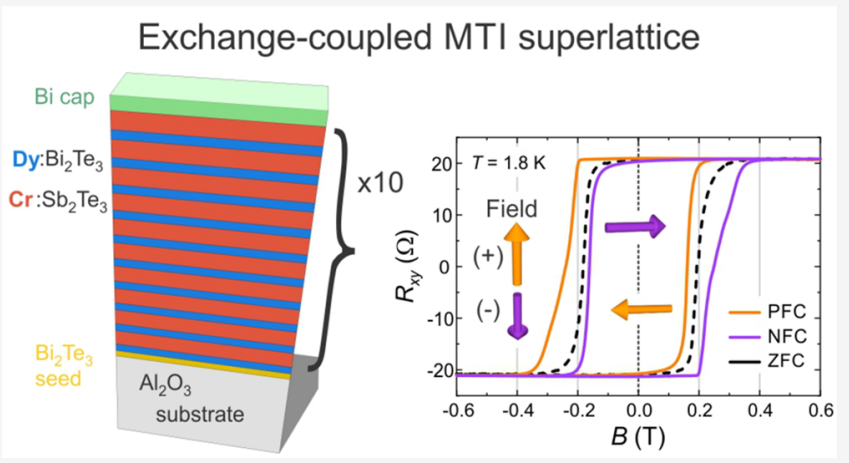
netic ordering in $\mathrm{Dy}: \mathrm{Bi}_{2} \mathrm{Te}_{3}$ induced by proximity coupling to $\mathrm{Cr}: \mathrm{Sb}_{2} \mathrm{Te}_{3}$ at the interface. This work provides a new pathway to realizing the quantum anomalous Hall effect at elevated temperatures and axion insulator state at zero magnetic field by interface engineering in TI heterostructures.

KEYWORDS: topological insulators, exchange bias, proximity effect, superlattice

$\mathrm{T}$ opological insulators (TIs) refer to materials that are electrically insulating in the bulk but possess topologically protected conducting surface states. Magnetic doping can break time-reversal symmetry (TRS) in TI materials and lead to the realization of the quantum anomalous Hall effect (QAHE) at low temperatures-a dissipationless transport phenomenon in the absence of an external magnetic field. ${ }^{1}$ The QAHE was first observed in $\mathrm{Cr}$-doped $(\mathrm{Bi}, \mathrm{Sb})_{2} \mathrm{Te}_{3}$ TI thin films, ${ }^{2}$ and afterward in $\mathrm{V}$-doped and $\mathrm{Cr}-\mathrm{V}$-codoped $(\mathrm{Bi}, \mathrm{Sb})_{2} \mathrm{Te}_{3}$ films. ${ }^{3,4}$ Despite the ferromagnetic (FM) ordering temperatures being $\sim 25 \mathrm{~K}$, the QAHE was only observed at low sub-K temperatures, which is attributed to Dirac-mass disorder, ${ }^{5}$ i.e., the fact that the inhomogeneous spatial distribution of magnetic dopants leads to spatially varying band gap sizes. To overcome the dilemma between dopant disorder (high doping scenario) and small band gap (low doping scenario), modulation doping has been applied to increase the QAHE temperature to $2 \mathrm{~K}^{6}$

Since the size of the band gap is directly proportional to the magnetic moment in TRS-broken TI materials, doping with rare earth (RE) elements such as Dy, which has a large atomic moment of up to $10.5 \mu_{\mathrm{B}}$, has been considered an alternative approach to raise the QAHE temperature. ${ }^{7}$ Contrary to most other RE dopants such as Gd and Ho, which show a constant atomic moment independent of doping concentration, ${ }^{8,9}$ a large atomic moment is found in $\mathrm{Dy}: \mathrm{Bi}_{2} \mathrm{Te}_{3}$ (DBT) films for low doping concentrations, thereby having the potential to be less detrimental to the electrical transport properties. ${ }^{10}$ This can be explained by possible antiferromagnetic (AFM) coupling between Dy atoms for higher concentrations, reminiscent of the behavior of elemental Dy. ${ }^{11}$ More importantly, inhomogeneous and short-ranged ferromagnetic patches have been revealed in DBT surrounded by a paramagnetic (PM) matrix. ${ }^{12}$ These findings bring Dy-doped TIs under the spotlight for exploring possible magnetic ordering by other means, one example being proximity coupling to ferromagnetic TI (MTI) layers such as $\mathrm{Cr}: \mathrm{Sb}_{2} \mathrm{Te}_{3}$ (CST). ${ }^{13}$

Here, we report the observation of an unexpected exchange bias effect in $[\mathrm{CST} / \mathrm{DBT}]_{10}$ superlattices, a topological insulator superlattice system with alternating magnetic transition-metal (TM)- and RE-doped layers. Utilizing both electrical transport and magneto-optical Kerr effect (MOKE) techniques, we find an exchange bias effect persisting up to 100 $\mathrm{K}$, with bias fields reaching $0.08 \mathrm{~T}$ at $1.8 \mathrm{~K}$. First-principles calculations indicate the presence of antiferromagnetic ordering in DBT layers close to the interfaces. Moreover, a significant increase of the magnetic ordering temperature is

Received: April 17, 2020

Revised: June 17, 2020

Published: June 18, 2020 

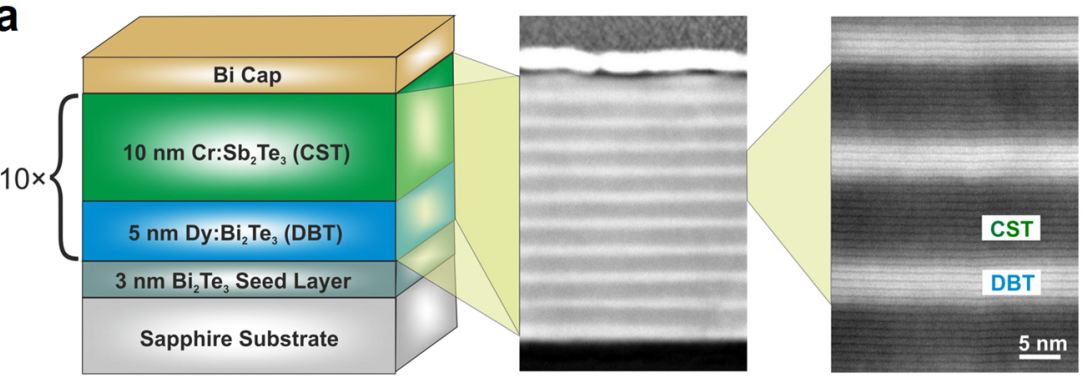

b

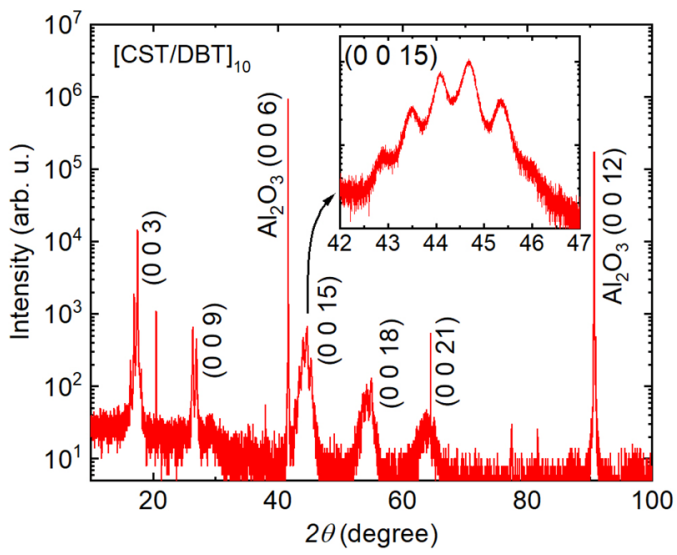

d

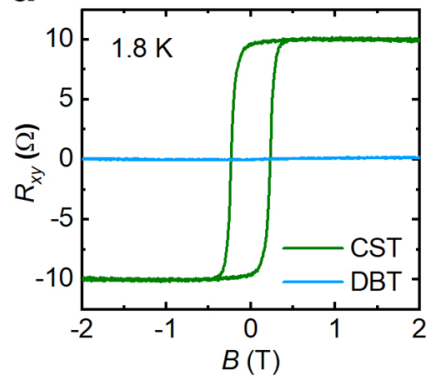

C

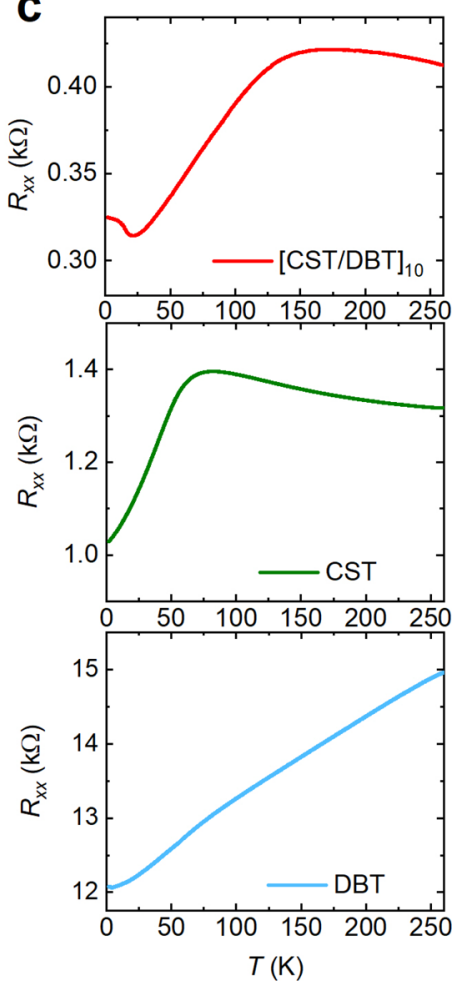

Figure 1. Structural properties of the $\mathrm{Cr}: \mathrm{Sb}_{2} \mathrm{Te}_{3} / \mathrm{Dy}_{\mathrm{Bi}} \mathrm{Be}_{3}$ superlattice. (a) Schematic of the $[\mathrm{CST} / \mathrm{DBT}]_{10}$ superlattice sample and corresponding cross-sectional HAADF-STEM images and, (b) XRD spectrum. The inset shows a detailed scan around the (0 015$)$ Bragg peak. (c) Temperaturedependent longitudinal resistance $\left(R_{x x}\right)$ measurements of the superlattice (red), $20 \mathrm{~nm}$ thick CST film (green), and $20 \mathrm{~nm}$ thick DBT film (blue). (d) Hall resistance $\left(R_{x y}\right.$; field applied normal to the film) of the CST (green) and DBT (blue) films at $1.8 \mathrm{~K}$. CST shows ferromagnetic behavior (out-of-plane easy axis), while DBT shows a paramagnetic response.
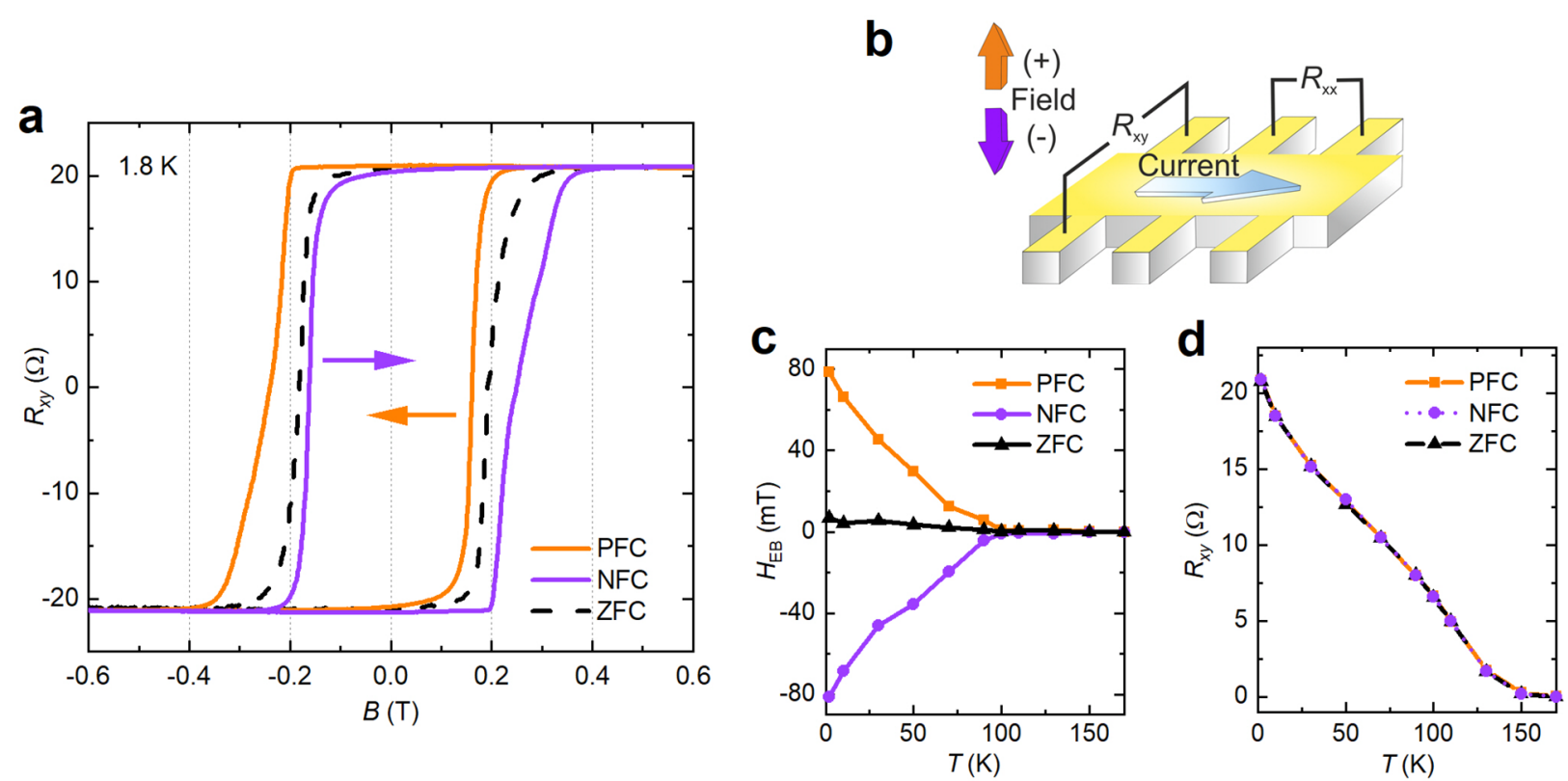

Figure 2. Temperature dependence of exchange bias in the TI superlattice. (a) Exchange-biased hysteresis loops of the $[\text { CST/DBT }]_{10}$ superlattice at $1.8 \mathrm{~K}$. The unbiased loop (black) from zero-field-cooling (ZFC) is shown as a reference. Positive-field-cooling (PFC) at $+6 \mathrm{~T}$ results in a shift of the unbiased loop to the left (orange), and negative-field-cooling (NFC) at $-6 \mathrm{~T}$ results in a shift to the right (purple). (b) Measurement geometry of the transport experiment with the field applied perpendicular to the film. (c, d) Temperature-dependent exchange bias field $\left(H_{\mathrm{EB}}\right)$ and $\mathrm{Hall}$ resistance $\left(R_{x y}\right)$, respectively, for the PFC, NFC, and ZFC cases.

found in the TI superlattice compared to a CST single-layer film. Our findings open the door to the rich physics of RE- doped TI materials and pave the way to future energy-efficient spintronic devices. 
The schematic of the $[\mathrm{CST} / \mathrm{DBT}]_{10}$ heterostructure sample and cross-sectional electron microscopy images are shown in Figure 1a. Starting with a $3 \mathrm{~nm}$ thick $\mathrm{Bi}_{2} \mathrm{Te}_{3}$ seed layer on a $c$ plane sapphire substrate, DBT $(5 \mathrm{~nm})$ and CST $(10 \mathrm{~nm})$ are alternately stacked to form 10-repeating bilayers, before terminating with a thin $\mathrm{Bi}$ cap. The nominal composition of the superlattice is $\left[\mathrm{Cr}_{0.41} \mathrm{Sb}_{1.59} \mathrm{Te}_{3} / \mathrm{Dy}_{0.62} \mathrm{Bi}_{1.38} \mathrm{Te}_{3}\right]_{10}$. As references, $20 \mathrm{~nm}$ thick CST and DBT single-layer films were grown and fabricated into microdevices using identical parameters as for the superlattice sample. The cross-sectional images of the superlattice sample were taken by high-angle annular dark-field scanning transmission electron microscopy (HAADF-STEM). ${ }^{13}$ In these images, the thinner, brighter layers are DBT, and the thicker, darker layers are CST. It can be seen that abrupt interfaces and constant layer thicknesses are well maintained throughout the stack. In the close-up, the quintuple layers (QLs) are clearly resolved in both CST and DBT which are separated by van der Waals gaps. Figure $1 \mathrm{~b}$ shows the X-ray diffraction (XRD) pattern of the superlattice. The presence of the series of $\left(\begin{array}{lll}0 & 0 & l\end{array}\right)$ peaks confirms the $c$-axis orientation and crystal structure. It is not possible to distinguish between $\mathrm{Sb}_{2} \mathrm{Te}_{3}$ and $\mathrm{Bi}_{2} \mathrm{Te}_{3}$ peaks in the spectrum since the lattice constants are very similar for doped samples $(c$ $\left.\approx 30.45 \AA^{10,14}\right)$. The superlattice peaks around the $\left(\begin{array}{lll}0 & 0 & 15\end{array}\right)$ reflection are shown in the inset, confirming the well-ordered superlattice seen in HAADF-STEM. Figure 1c shows the temperature-dependent longitudinal resistance $\left(R_{x x}\right)$ of the superlattice and single-layer films of CST and DBT. Both the superlattice and CST film experience a magnetic phase transition at about 150 and $80 \mathrm{~K}$, respectively, which is reflected by the maximum in resistance. However, no magnetic phase transition was observed in the $R_{x x}$ of the DBT film. Figure $1 \mathrm{~d}$ shows the Hall resistance $\left(R_{x y}\right)$ of CST and DBT films measured at the base temperature of the cryostat $(1.8 \mathrm{~K})$. A square-shaped hysteresis was found in CST, demonstrating ferromagnetic ordering with an easy axis pointing out-of-plane. In comparison, DBT does not exhibit magnetic order in the perpendicular direction, matching our previous finding that DBT on its own is paramagnetic. ${ }^{10,12}$

The exchange bias effect in the $[\mathrm{CST} / \mathrm{DBT}]_{10}$ superlattice at $1.8 \mathrm{~K}$ is shown in Figure $2 \mathrm{a}$, where the hysteresis loops are shifted opposite to the direction of the field applied during cooling. This measurement is conducted on a microsized Hall bar after positive-field-cooling (PFC) and negative-fieldcooling (NFC) from $300 \mathrm{~K}$, with the field applied perpendicular to the film (Figure $2 \mathrm{~b}$ ). When a field of $+6 \mathrm{~T}$ is applied during cooling (orange up-arrow in Figure $2 b$ ), the hysteresis loop shows a horizontal shift toward the left direction (orange loop in Figure 2a), and a cooling field of $-6 \mathrm{~T}$ (purple down-arrow in Figure 2b) induces a shift toward the right (purple loop in Figure 2a). The descending branches (orange and purple loops) show a steep drop while the ascending branches are more rounded. This asymmetry is a common characteristic in exchange-biased systems such as $\mathrm{Co} /$ $\mathrm{CoO}$ nanoparticles. ${ }^{15}$ For reference, Figure $2 \mathrm{a}$ also includes the hysteresis loop after zero-field-cooling (ZFC, black loop). The exchange bias effect can be observed up to $100 \mathrm{~K}$ (see Figure S1 in the Supporting Information). The exchange bias field $\left(H_{\mathrm{EB}}\right)$, defined as the shift of the center of the hysteresis loop from the unbiased case, reaches $0.08 \mathrm{~T}$ at $1.8 \mathrm{~K}$ and decreases monotonically with increasing temperature (Figure 2c). The spontaneous magnetization also follows a decreasing trend as temperature increases and approaches 0 at 130-150 K (Figure
$2 \mathrm{~d})$, marking the Curie temperature $\left(T_{\mathrm{C}}\right)$ of the superlattice system. This value matches the temperature for the magnetic phase transition revealed in Figure 1c. Notably, we can also find similar results of exchange bias and temperaturedependent magnetization of the superlattice, using superconducting quantum interference device (SQUID) magnetometry (see Figure S2 in the Supporting Information). In some materials, a full magnetization reversal can contain two steps, meaning that an intermediate plateau may exist in the middle of the hysteresis loop. A field sweep range that terminates at the plateau can then result in a horizontally shifted loop with a reduced loop height. ${ }^{16}$ Here, we do not find a difference in the magnetic response for the three cases (orange, purple, and black loops) over the entire temperature range, nor did we see evidence for minor loops outside the $\pm 0.6 \mathrm{~T}$ range (in fields up to $\pm 8 \mathrm{~T}$ ). We can therefore conclude that the exchange bias effect in $[\mathrm{CST} / \mathrm{DBT}]_{10}$ is robust in loops cycling up to $\pm 8 \mathrm{~T}$.

To further explore the temperature dependence of the exchange bias in the superlattice, we conducted polar MOKE experiments using the geometry shown in Figure $3 a,{ }^{17}$ where a linearly polarized laser beam is perpendicularly incident on the sample and reflected with a small polarization rotation angle. The experimental procedure follows the step-by-step diagram shown in Figure $3 \mathrm{~b}$. The sample was first zero-field-cooled from $300 \mathrm{~K}$ to a poling temperature $T_{\mathrm{A}}$. At this temperature, a $+6 \mathrm{~T}$ field was applied to the film and then reduced again to 0 . Subsequently, the film was zero-field-cooled to $10 \mathrm{~K}$, and the MOKE hysteresis loops were recorded $( \pm 0.6 \mathrm{~T}$ field sweep). After the completion of this measurement, the sample was zero-field-warmed (ZFW) to $300 \mathrm{~K}$ and the same cooling process was repeated for a different temperature $T_{\mathrm{A}}$. The hysteresis loops with varying $T_{\mathrm{A}}$ are shown in Figure $3 \mathrm{c}$, and the corresponding exchange bias field is plotted in Figure 3d. Clearly, when $T_{\mathrm{A}}$ is increased from 90 to $170 \mathrm{~K}$ the loops are shifting gradually from maximum bias to the unbiased state. The results obtained with $T_{\mathrm{A}} \geq 140 \mathrm{~K}$ are identical to the ZFC case from $300 \mathrm{~K}$, and the results with $T_{\mathrm{A}} \leq 100 \mathrm{~K}$ are identical to the $+6 \mathrm{~T}$ PFC case (see Figure $\mathrm{S} 3$ in the Supporting Information). The explanation of this behavior is that below $140 \mathrm{~K}, \mathrm{CST}$ layers are ferromagnetically ordered such that by applying an out-of-plane field, a perpendicular remanent magnetization is established. However, below $100 \mathrm{~K}$, exchange bias results from the coupling between the ferromagnetic CST layer and an apparently antiferromagnetic DBT layer at the interface. To conclude the experimental findings, the ferromagnetic response of the superlattice has a Curie temperature of $\sim 140 \mathrm{~K}$, and the exchange bias effect is present below $100 \mathrm{~K}$.

The paramagnetic nature of DBT makes the exchange bias in $[\mathrm{CST} / \mathrm{DBT}]_{10}$ a unique phenomenon, since this effect is observed in FM/AFM heterostructures but not in FM/PM systems. In FM/AFM heterostructures, the spins of the FM layer are pinned by the AFM layer via an exchange interaction across the interface. Even though several Cr- or Dy-based compounds are known to be antiferromagnetic, such as $\mathrm{Cr}_{2} \mathrm{O}_{3}$ and $\mathrm{Dy}_{2} \mathrm{Te}_{3}$, there is no secondary phase observed in the XRD spectrum or in the HAADF-STEM images of the TI superlattice that could support an AFM coupling scenario. It should be noted though that the existence of exchange bias in $\mathrm{FM} / \mathrm{PM}$ systems is not unprecedented; , one example being $\mathrm{LaMnO}_{3} / \mathrm{LaNiO}_{3}$ superlattices examined by Gibert et al. ${ }^{18}$ The authors ascribe the ferromagnetic interfacial coupling between $\mathrm{Mn}$ and $\mathrm{Ni}$ to the close lattice matching between the 

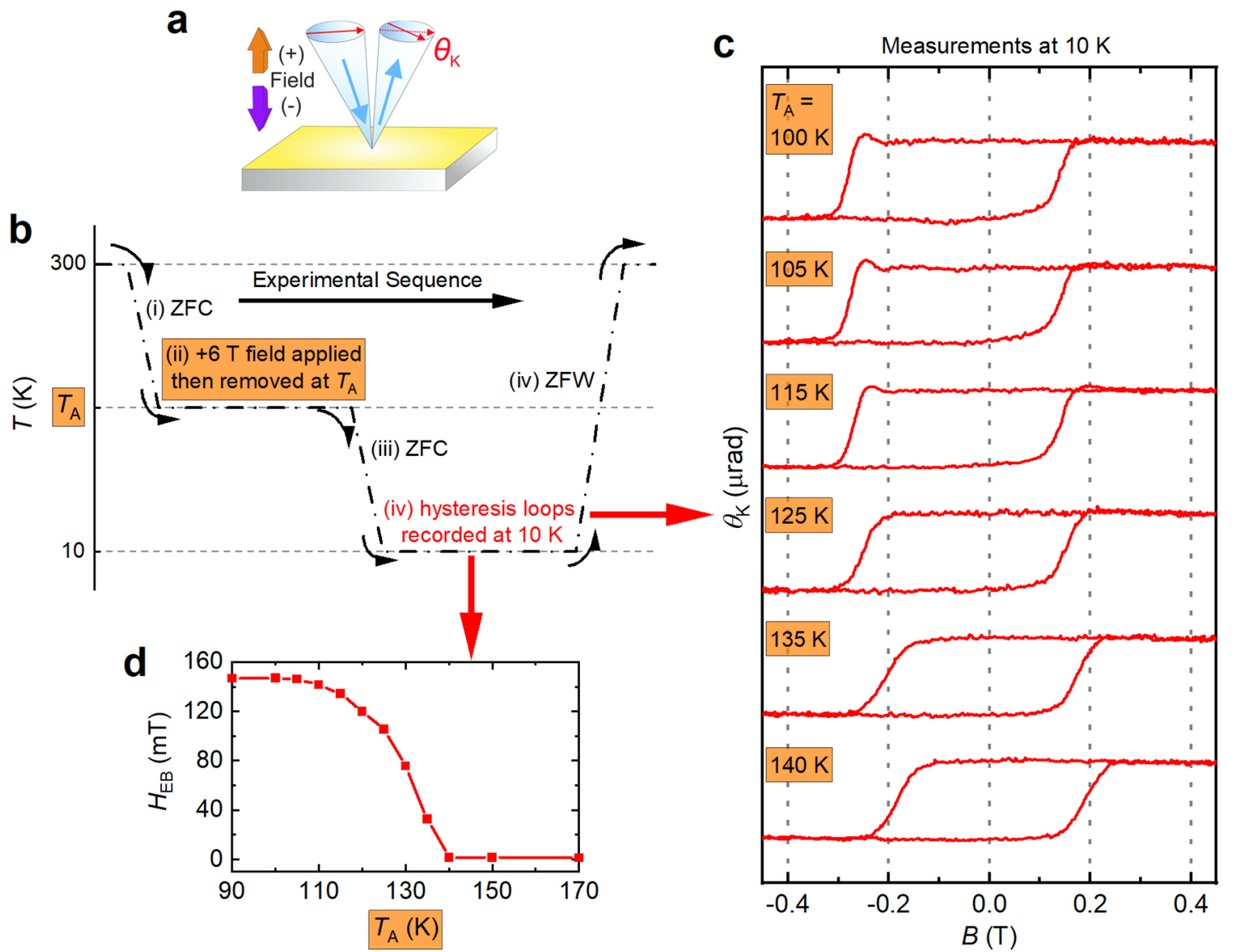

Figure 3. Exchange bias induced by applying a magnetic field at high temperatures. (a) Measurement geometry of the polar MOKE experiment. (b) Illustration of the experimental sequence: (i) First, the sample is zero-field-cooled (ZFC) from $300 \mathrm{~K}$ down to a poling temperature, $T_{\mathrm{A}}$. (ii) At $T_{\mathrm{A}}$, a field of $+6 \mathrm{~T}$ is applied and then reduced to 0. (iii), (iv) After ZFC down to $10 \mathrm{~K}$, the hysteresis loops are recorded. (v) Finally, the sample is zero-field-warmed $(\mathrm{ZFW})$ to $300 \mathrm{~K}$. (c) Polar MOKE hysteresis loops at $10 \mathrm{~K}$ for different poling temperatures $T_{\mathrm{A}}$. (d) Exchange bias field as a function of the poling temperature $T_{\mathrm{A}}$.

layers and to induced magnetic ordering in $\mathrm{LaNiO}_{3}$ that resembles a spin-density wave. Considering the structural similarity between the CST and DBT layers, it can be expected that an interfacial effect may arise in the TI superlattice akin to $\mathrm{LaMnO}_{3} / \mathrm{LaNiO}_{3}$. Apart from the lattice matching, the exchange bias effect can also be linked to the interface roughness in a heterostructure. ${ }^{19,20}$ It has been reported that an interface roughness of $1-2 \mathrm{~nm}$ in $\mathrm{Co} / \mathrm{CoO}$ bilayers can strengthen the exchange bias field as more uncompensated antiferromagnetic spins are induced by structural defects that couple to the ferromagnetic layer. ${ }^{21}$ Our electron microscopy data reveal an interface roughness between the $\mathrm{Cr}$ - and Dydoped layers on the level of $1 \mathrm{QL}$, which may also play a role in the interfacial exchange coupling.

In order to understand the interfacial coupling between CST and DBT in more detail, we carried out density functional theory (DFT) calculations. The calculated lattice constants for hexagonal $\mathrm{Sb}_{2} \mathrm{Te}_{3}$ and $\mathrm{Bi}_{2} \mathrm{Te}_{3}$ are $a=4.33 \AA, c=31.34 \AA$, and $a$ $=4.44 \AA, c=31.91 \AA$, respectively, which agree well with the established experimental values for bulk crystals. ${ }^{22}$ In the TI superlattice, our calculations show that the $\mathrm{Cr}$ and Dy spins closest to the interface tend to align antiparallelly to each other, agreeing with the observations made in many TM/RE magnetic heterostructures. ${ }^{23,24}$ When two layers of Dy atoms at the interface are considered, and the $\mathrm{Cr}$ spins are all aligned in the "up" direction, the energetically most stable state of the $\mathrm{Cr}-\mathrm{Dy}-\mathrm{Dy}$ chain is the "up-down-up" configuration. This means the two Dy layers prefer to locally order antiferromagnetically, making the CST/DBT heterostructure effectively an interfacial FM/AFM system-the common scenario for inducing exchange bias. Figure 4 a shows an atomic spin model of $\mathrm{Cr}$ and Dy close to the interface during an exchange-biased magnetization reversal after positive-field-cooling. The calculations show that $\mathrm{Cr}$ and $\mathrm{Dy}$ atoms possess magnetic moments of $3.4 \mu_{\mathrm{B}}$ and $4.7 \mu_{\mathrm{B}}$, respectively, in line with previous experimental results. ${ }^{10,14}$

The density of states (DOS) of the TI superlattice in the "up-down-up" state is shown in Figure 4b. As can be seen in the DOS plot, the $\mathrm{Cr}$ dopants contribute conduction carriers to the superlattice structure and thus raise its Fermi level $\left(E_{\mathrm{F}}\right)$ toward the conduction band, i.e., making the whole sample metallic (increasing the conductivity). In contrast, the distribution of Dy states is mainly concentrated at around $-22 \mathrm{eV}$ with only a small portion close to the Fermi level, indicating that Dy dopants have a marginal effect on the conductivity of the superlattice sample. This finding echoes the high-resistance results of the DBT film shown in Figure 1c.

Figure 5 shows the temperature dependence of the magnetization in a zero-field-cooled $[\mathrm{CST} / \mathrm{DBT}]_{10}$ superlattice and a CST film of the same nominal doping concentration, using the experimental geometry depicted in Figure 3a. Both the superlattice (Figure 5a) and the single-layer film (Figure 5b) show similar square-shaped hysteresis loops, which is not surprising as the ferromagnetism of the superlattice originates 
a

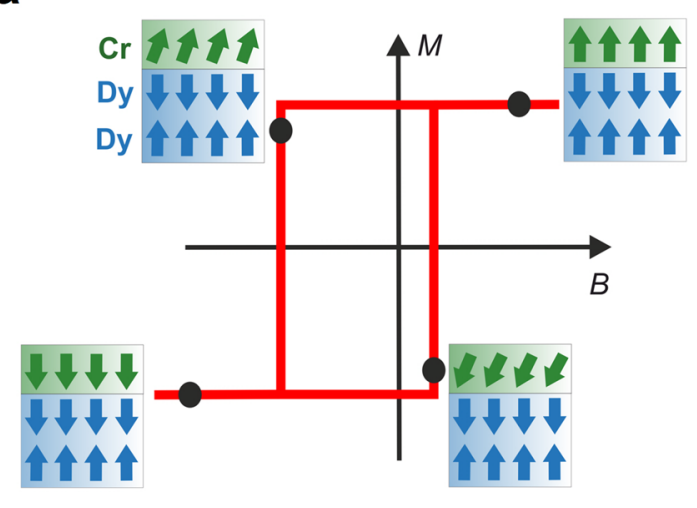

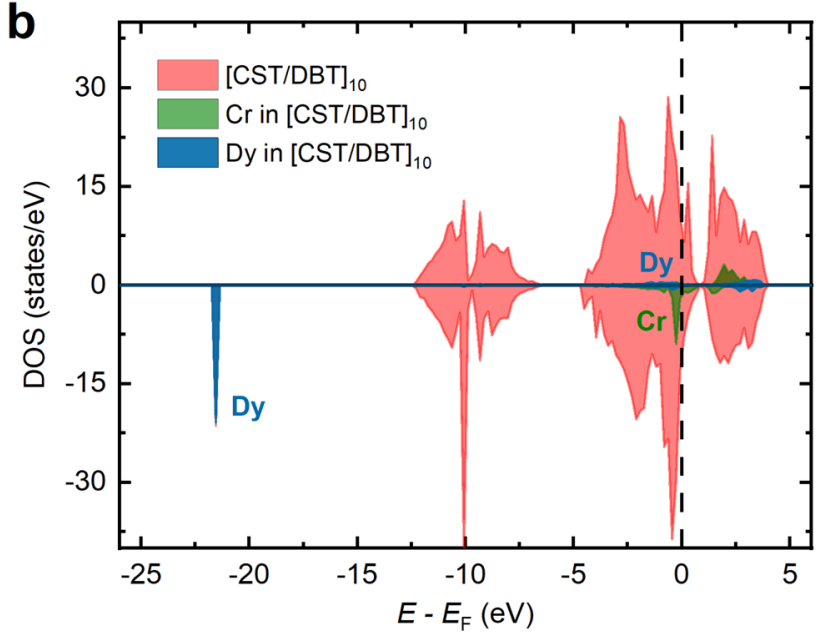

Figure 4. Results of the first-principles calculations. (a) Schematic of the atomic spin model at the interfaces of $[\mathrm{CST} / \mathrm{DBT}]_{10}$, in a biased hysteresis loop after positive-field-cooling. The cooling field aligns the spins of $\mathrm{Cr}-\mathrm{Dy}-\mathrm{Dy}$ to the "up-down-up" configuration. The Dy atoms stay antiferromagnetically ordered throughout the magnetization reversal of the $\mathrm{Cr}$ atoms. (b) Density of states (DOS) of $[\mathrm{CST} / \mathrm{DBT}]_{10}$ in the "up-down-up" configuration, illustrating the DOS contributions from Cr (green), Dy (blue), and the entire material (red).
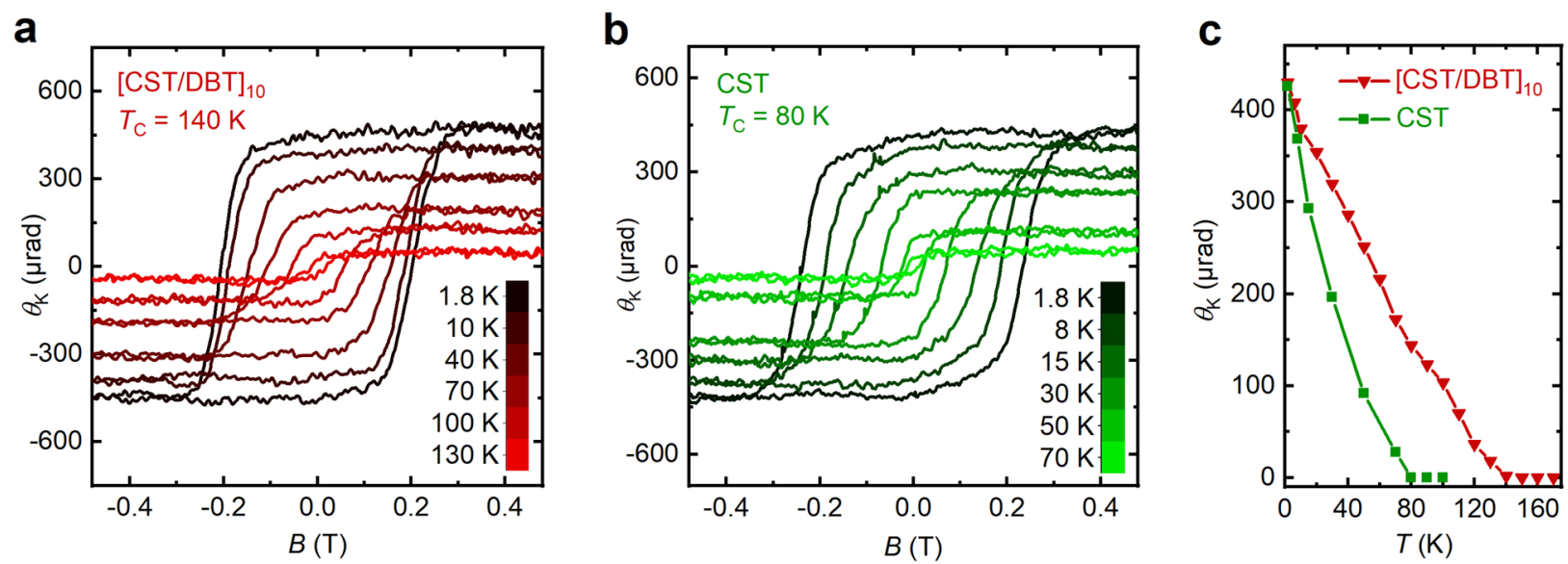

Figure 5. Temperature-dependent ferromagnetism in the TI superlattice and single-layer film. (a) Polar MOKE hysteresis loops of the [CST/ $\mathrm{DBT}]_{10}$ superlattice for a range of temperatures. Results are obtained after zero-field-cooling. (b) Corresponding hysteresis loops of the CST film. (c) Comparison of the temperature dependence of the magnetization of the two samples. The out-of-plane ferromagnetic ordering in [CST/ $\mathrm{DBT}_{10}$ (red) and CST (green) disappears at 140 and $80 \mathrm{~K}$, respectively.

from the Cr-doped layers. The main difference lies in the temperature dependence, highlighted in Figure 5c. While the CST single-layer film loses its magnetic order when approaching $80 \mathrm{~K},{ }^{25}$ taken as the Curie temperature of the film, the superlattice preserves its ferromagnetism up to $\sim 140$ $\mathrm{K}$, i.e., reaching almost twice the $T_{\mathrm{C}}$ of CST itself.

The observation of an increased $T_{C}$ in the superlattice is tied in closely to the AFM nature of DBT at the interface. It has been identified in several FM/AFM heterostructures that exchange coupling to an AFM can provide extra uniaxial anisotropy, and therefore increase the Curie temperature of the FM layer ${ }^{26}$ - not only in conventional FM materials like $\mathrm{FeMn} / \mathrm{Co}^{27,28}$ but also in MTI systems such as $\mathrm{Cr}$ : $(\mathrm{Bi}, \mathrm{Sb})_{2} \mathrm{Te}_{3} / \mathrm{CrSb}$ and $\mathrm{Cr}: \mathrm{Sb}_{2} \mathrm{Te}_{3} / \mathrm{Cr}_{2} \mathrm{O}_{3} \cdot{ }^{29,30}$ By increasing the thickness of the antiferromagnetic $\mathrm{Cr}_{2} \mathrm{O}_{3}$ layer, the Curie temperature of $\mathrm{Cr}: \mathrm{Sb}_{2} \mathrm{Te}_{3} / \mathrm{Cr}_{2} \mathrm{O}_{3}$ increases from 39 to $50 \mathrm{~K}$ compared to the $\mathrm{Cr}: \mathrm{Sb}_{2} \mathrm{Te}_{3}$ single-layer film. ${ }^{30}$ More interestingly, by tuning the magnitude of the cooling field, both positive and negative exchange bias effects have been observed in the heterostructure, demonstrating the potential to further develop TI-based spintronic devices through manipulation of the exchange bias. Regarding $\mathrm{Cr}$ : $(\mathrm{Bi}, \mathrm{Sb})_{2} \mathrm{Te}_{3} / \mathrm{CrSb}$, He et al. demonstrated a monotonic increase of $T_{\mathrm{C}}$ from 40 to $90 \mathrm{~K}$ when the MTI/AFM superlattice [Cr: $(\mathrm{Bi}, \mathrm{Sb})_{2} \mathrm{Te}_{3} /$ $\mathrm{CrSb}]_{n}$ is stacked up from $n=0$ (only Cr-doped layer) to $n=$ $10{ }^{29}$ A possible explanation of this phenomenon is the influence of strain on the magnetocrystalline anisotropy. As the DBT layers have a $3 \%$ lattice mismatch with the CST layers, tensile strain can accumulate in the 10-repeat CST/DBT sample and modulate the magnetocrystalline anisotropy of CST in the system, pushing up the transition temperature. Similar strain-tuning effects of $T_{\mathrm{C}}$ have also been reported in $[\mathrm{La}, \mathrm{Sr}] \mathrm{MnO}_{3}$ films and $[\mathrm{La}, \mathrm{Sr}] \mathrm{MnO}_{3} / \mathrm{BaTiO}_{3}$ superlattices. $^{31,32}$

One of the ultimate goals for MTIs is to achieve the QAHE at room temperature. However, the current record is $\sim 2 \mathrm{~K}$ despite a magnetic transition temperature of $\sim 250 \mathrm{~K}^{6,33}$ The reason for this discrepancy may lie in the inhomogeneity of the magnetic dopants and thus the electronic properties. ${ }^{5}$ Proximity-coupled undoped-TI/ferromagnetic-insulator (TI/ 
FI) heterostructures, in which dopants and current-carrying layers are spatially separated, are therefore another viable route. ${ }^{34-36}$ However, the anomalous Hall resistance in TI/FI heterostructures is far from the quantum regime, possibly due to the weak exchange coupling between FI and TI across the interface of the dissimilar lattice structures. Apart from TI/FI systems, proximity coupling to an AFM layer, such as latticematched $\mathrm{CrSb}$, MnTe, or $\mathrm{Cr}_{2} \mathrm{O}_{3}$, can also enhance the ferromagnetic order in MTI/AFM heterostructures. ${ }^{29,30,37}$ However, one limiting factor is that the magnetism of the binary AFM is a given materials property and can only be to some degree tuned via the film thickness.

However, Dy-doped TIs inherently provide more freedom to optimize the MTI/AFM system, in terms of both doping level and thickness control. The ideal lattice matching between CST and DBT also ensures a strong exchange coupling between the two layers. This means TM/RE-doped superlattices may work as an alternative path for increasing the QAHE temperature. Furthermore, by optimizing the growth and structure of the TI film, it may be possible to create a strongly biased Cr-/Dy-doped heterostructure with the hysteresis loop fully shifted to one side. Integrating this with a nonbiased MTI layer can create an axion insulator state that persists without an external magnetic field, unlocking topological magnetoelectric effects. ${ }^{38,39}$

Apart from the exchange bias, the combination of TIs and antiferromagnetism offers a fruitful platform for exploring other effects of nontrivial topology, either through TI/AFM heterostructure engineering or through establishing antiferromagnetic order in intrinsic MTI materials. For instance, the seeding of topological charges has been observed in $(\mathrm{Bi}, \mathrm{Sb})_{2} \mathrm{Te}_{3} / \mathrm{MnTe}$ heterostructures ascribed to interfacial spin pinning, ${ }^{37}$ leading to the topological Hall effect-a signature of real-space chiral spin textures. Further, a recent study has confirmed the realization of QAHE on $\mathrm{MnBi}_{2} \mathrm{Te}_{4}$ thin flakes, an intrinsic MTI material with antiferromagnetic ordering. ${ }^{40}$ By proposing an experimental approach combining AFM-ordered TIs and heterostructure engineering, it has therefore been predicted that, $\mathrm{MnBi}_{2} \mathrm{Te}_{4} / \mathrm{CrI}_{3}$ heterostructure films can host both the QAHE and exchange bias, which preserves an out-of-plane surface magnetism through proximity coupling. ${ }^{41}$

In summary, we unambiguously identified an exchange bias effect in the Cr-/Dy-doped TI superlattice $[\mathrm{CST} / \mathrm{DBT}]_{10}$ using both magneto-optical and magneto-transport techniques. The onset temperature of the exchange bias effect is $\sim 100 \mathrm{~K}$, with the ferromagnetic ordering persisting up to $140 \mathrm{~K}$. This Curie temperature in the superlattice is enhanced by $60 \mathrm{~K}$ compared to a Cr-doped single-layer film. First-principles calculations suggest that the origin of the exchange bias effect lies in the antiferromagnetic ordering of Dy close to the interface, i.e., in close proximity to Cr. Our findings highlight the wealth of tuning possibilities in tailored TI heterostructures, opening new avenues for achieving high-temperature QAHE and zero-field topological magnetoelectric effect in exchange-biased magnetic systems.

\section{ASSOCIATED CONTENT}

\section{s) Supporting Information}

The Supporting Information is available free of charge at https://pubs.acs.org/doi/10.1021/acs.nanolett.0c01666.
Methods, exchange-biased hysteresis loops of the TI superlattice at different temperatures probed by electrical transport measurements, exchange-biased magnetic response of the TI superlattice probed by SQUID magnetometry, and exchange-biased hysteresis loops of the TI superlattice at different temperatures probed by MOKE measurements (PDF)

\section{AUTHOR INFORMATION}

\section{Corresponding Authors}

Jieyi Liu - Clarendon Laboratory, Department of Physics, University of Oxford, Oxford OX1 3PU, United Kingdom; Cavendish Laboratory, University of Cambridge, Cambridge CB3 OHE, United Kingdom; Email: Jieyi.Liu@ physics.ox.ac.uk

Thorsten Hesjedal - Clarendon Laboratory, Department of Physics, University of Oxford, Oxford OX1 3PU, United Kingdom; 이이이.org/0000-0001-7947-3692; Email: Thorsten.Hesjedal@physics.ox.ac.uk

\section{Authors}

Angadjit Singh - Cavendish Laboratory, University of Cambridge, Cambridge CB3 OHE, United Kingdom; Department of Physics, Royal Holloway, University of London, Egham TW20 OEX, United Kingdom

Yu Yang Fredrik Liu - Cavendish Laboratory, University of Cambridge, Cambridge CB3 OHE, United Kingdom

Adrian Ionescu - Cavendish Laboratory, University of Cambridge, Cambridge CB3 OHE, United Kingdom

Balati Kuerbanjiang - Clarendon Laboratory, Department of Physics, University of Oxford, Oxford OX1 3PU, United Kingdom

Crispin H. W. Barnes - Cavendish Laboratory, University of Cambridge, Cambridge CB3 OHE, United Kingdom

Complete contact information is available at:

https://pubs.acs.org/10.1021/acs.nanolett.0c01666

\section{Author Contributions}

J.L. and A.S. contributed equally to this work.

\section{Notes}

The authors declare no competing financial interest.

\section{ACKNOWLEDGMENTS}

J.L. and T.H. acknowledge financial support from EPSRC (Grant No. EP/M020517/1). A.S. acknowledges financial support from the SGPC Cambridge Commonwealth Trust, and C.H.W.B. is grateful for financial support from EPSRC (Grant No. EP/J00412X/1). T.H. and B.K. acknowledge financial support from the John Fell Oxford University Press Research Fund and EPSRC (Grant No. EP/N032128/1). We thank Dr. Liam B. Duffy for help with the sample growth.

\section{REFERENCES}

(1) Yu, R.; Zhang, W.; Zhang, H.-J.; Zhang, S.-C.; Dai, X.; Fang, Z. Quantized anomalous Hall effect in magnetic topological insulators. Science 2010, 329, 61-64.

(2) Chang, C.-Z.; et al. Experimental observation of the quantum anomalous Hall effect in a magnetic topological insulator. Science 2013, 340, 167-170.

(3) Chang, C.-Z.; Zhao, W.; Kim, D. Y.; Zhang, H.; Assaf, B. A.; Heiman, D.; Zhang, S. C.; Liu, C.; Chan, M. H. W.; Moodera, J. S. High-precision realization of robust quantum anomalous Hall state in 
a hard ferromagnetic topological insulator. Nat. Mater. 2015, 14, 473-477.

(4) Ou, Y.; et al. Enhancing the quantum anomalous Hall effect by magnetic codoping in a topological insulator. Adv. Mater. 2018, 30, 1703062.

(5) Lee, I.; Kim, C. K.; Lee, J.; Billinge, S. J. L.; Zhong, R.; Schneeloch, J. A.; Liu, T.; Valla, T.; Tranquada, J. M.; Gu, G.; Davis, J. C. S. Imaging Dirac-mass disorder from magnetic dopant atoms in the ferromagnetic topological insulator $\mathrm{Cr}_{x}\left(\mathrm{Bi}_{0.1} \mathrm{Sb}_{0.9}\right)_{2-x} \mathrm{Te}_{3}$. Proc. Natl. Acad. Sci. U. S. A. 2015, 112, 1316-1321.

(6) Mogi, M.; Yoshimi, R.; Tsukazaki, A.; Yasuda, K.; Kozuka, Y.; Takahashi, K. S.; Kawasaki, M.; Tokura, Y. Magnetic modulation doping in topological insulators toward higher-temperature quantum anomalous Hall effect. Appl. Phys. Lett. 2015, 107, 182401.

(7) Hesjedal, T. Rare Earth Doping of Topological Insulators: A Brief Review of Thin Film and Heterostructure Systems. Phys. Status Solidi A 2019, 216, 1800726.

(8) Harrison, S. E.; Collins-McIntyre, L. J.; Li, S.; Baker, A. A.; Shelford, L. R.; Huo, Y.; Pushp, A.; Parkin, S. S. P.; Harris, J. S.; Arenholz, E.; van der Laan, G.; Hesjedal, T. Study of Gd-doped $\mathrm{Bi}_{2} \mathrm{Te}_{3}$ thin films: Molecular beam epitaxy growth and magnetic properties. J. Appl. Phys. 2014, 115, No. 023904.

(9) Harrison, S. E.; Collins-McIntyre, L. J.; Zhang, S. L.; Baker, A. A.; Figueroa, A. I.; Kellock, A. J.; Pushp, A.; Chen, Y. L.; Parkin, S. S. P.; Harris, J. S.; van der Laan, G.; Hesjedal, T. Study of Ho-doped $\mathrm{Bi}_{2} \mathrm{Te}_{3}$ topological insulator thin films. Appl. Phys. Lett. 2015, 107, 182406.

(10) Harrison, S. E.; Collins-McIntyre, L. J.; Zhang, S. L.; Baker, A. A.; Figueroa, A. I.; Kellock, A. J.; Pushp, A.; Parkin, S. S. P.; Harris, J. S.; van der Laan, G.; Hesjedal, T. Study of Dy-doped $\mathrm{Bi}_{2} \mathrm{Te}_{3}$ : thin film growth and magnetic properties. J. Phys.: Condens. Matter 2015, 27, 245602.

(11) Behrendt, D.; Legvold, S.; Spedding, F. Magnetic properties of dysprosium single crystals. Phys. Rev. 1958, 109, 1544.

(12) Duffy, L. B.; et al. Microscopic effects of Dy doping in the topological insulator $\mathrm{Bi}_{2} \mathrm{Te}_{3}$. Phys. Rev. B: Condens. Matter Mater. Phys. 2018, 97, 174427.

(13) Duffy, L. B.; Steinke, N.-J.; Burn, D. M.; Frisk, A.; Lari, L.; Kuerbanjiang, B.; Lazarov, V. K.; van der Laan, G.; Langridge, S.; Hesjedal, T. Magnetic profile of proximity-coupled (Dy,Bi) ${ }_{2} \mathrm{Te}_{3} /$ $(\mathrm{Cr}, \mathrm{Sb})_{2} \mathrm{Te}_{3}$ topological insulator heterostructures. Phys. Rev. B: Condens. Matter Mater. Phys. 2019, 100, No. 054402.

(14) Collins-Mcintyre, L. J.; Duffy, L. B.; Singh, A.; Steinke, N. J.; Kinane, C. J.; Charlton, T. R.; Pushp, A.; Kellock, A. J.; Parkin, S. S.; Holmes, S. N.; Barnes, C. H. W.; van der Laan, G.; Langridge, S.; Hesjedal, T. Structural, electronic, and magnetic investigation of magnetic ordering in MBE-grown $\mathrm{Cr}_{x} \mathrm{Sb}_{2-x} \mathrm{Te}_{3}$ thin films. EPL 2016, $115,27006$.

(15) Hu, Y.; Liu, Y.; Du, A.; Shi, F. Dependence of exchange bias on core/shell relative dimension in ferromagnetic/antiferromagnetic nanoparticles. Phys. Lett. A 2014, 378, 1667-1674.

(16) Geshev, J. Comment on:"Exchange bias and vertical shift in $\mathrm{CoFe}_{2} \mathrm{O}_{4}$ nanoparticles" [J. Magn. Magn. Mater. 313 (2007) 266]. J. Magn. Magn. Mater. 2008, 320, 600-602.

(17) Liu, J.; Singh, A.; Llandro, J.; Duffy, L. B.; Stanton, M. R.; Holmes, S. N.; Applegate, M. J.; Phillips, R. T.; Hesjedal, T.; Barnes, C. H. W. A low-temperature Kerr effect microscope for the simultaneous magneto-optic and magneto-transport study of magnetic topological insulators. Meas. Sci. Technol. 2019, 30, 125201.

(18) Gibert, M.; Zubko, P.; Scherwitzl, R.; Íñiguez, J.; Triscone, J.M. Exchange bias in $\mathrm{LaNiO}_{3}-\mathrm{LaMnO}_{3}$ superlattices. Nat. Mater. 2012, 11, 195-198.

(19) Nogués, J.; Lederman, D.; Moran, T.; Schuller, I. K.; Rao, K. Large exchange bias and its connection to interface structure in $\mathrm{FeF}_{2}-$ Fe bilayers. Appl. Phys. Lett. 1996, 68, 3186-3188.

(20) Vafaee, M.; Finizio, S.; Deniz, H.; Hesse, D.; Zabel, H.; Jakob, G.; Kläui, M. The effect of interface roughness on exchange bias in $\mathrm{La}_{0.7} \mathrm{Sr}_{0.3} \mathrm{MnO}_{3}-\mathrm{BiFeO}_{3}$ heterostructures. Appl. Phys. Lett. 2016, 108, No. 072401.
(21) Wang, J.; Sannomiya, T.; Shi, J.; Nakamura, Y. Influence of interface roughness on the exchange bias of $\mathrm{Co} / \mathrm{CoO}$ multilayers. J. Appl. Phys. 2013, 113, 17D707.

(22) Yavorsky, B. Y.; Hinsche, N.; Mertig, I.; Zahn, P. Electronic structure and transport anisotropy of $\mathrm{Bi}_{2} \mathrm{Te}_{3}$ and $\mathrm{Sb}_{2} \mathrm{Te}_{3}$. Phys. Rev. B: Condens. Matter Mater. Phys. 2011, 84, 165208.

(23) Shan, Z.; Sellmyer, D. J. Magnetism of rare-earth-transitionmetal nanoscale multilayers. I. Experiments on Dy/Co, Dy/Fe, and Tb/Fe. Phys. Rev. B: Condens. Matter Mater. Phys. 1990, 42, 10433.

(24) Cherifi, K.; Dufour, C.; Bauer, P.; Marchal, G.; Mangin, P. Experimental magnetic phase diagram of a $\mathrm{Gd} / \mathrm{Fe}$ multilayered ferrimagnet. Phys. Rev. B: Condens. Matter Mater. Phys. 1991, 44, $7733-7736$.

(25) Singh, A.; Kamboj, V. S.; Liu, J.; Llandro, J.; Duffy, L. B.; Senanayak, S. P.; Beere, H. E.; Ionescu, A.; Ritchie, D. A.; Hesjedal, T.; Barnes, C. H. W. Systematic study of ferromagnetism in $\mathrm{Cr}_{x} \mathrm{Sb}_{2-x} \mathrm{Te}_{3}$ topological insulator thin films using electrical and optical techniques. Sci. Rep. 2018, 8, 17024.

(26) Sort, J.; Garcia, F.; Auffret, S.; Rodmacq, B.; Dieny, B.; Langlais, V.; Suriñach, S.; Muñoz, J.; Baró, M.; Nogués, J. Using exchange bias to extend the temperature range of square loop behavior in $[\mathrm{Pt} / \mathrm{Co}]$ multilayers with perpendicular anisotropy. Appl. Phys. Lett. 2005, 87, 242504.

(27) Won, C.; Wu, Y.; Zhao, H.; Scholl, A.; Doran, A.; Kim, W.; Owens, T.; Jin, X.; Qiu, Z. Studies of $\mathrm{FeMn} / \mathrm{Co} / \mathrm{Cu}(001)$ films using photoemission electron microscopy and surface magneto-optic Kerr effect. Phys. Rev. B: Condens. Matter Mater. Phys. 2005, 71, No. 024406.

(28) Lenz, K.; Zander, S.; Kuch, W. Magnetic proximity effects in antiferromagnet/ferromagnet bilayers: The impact on the Néel temperature. Phys. Rev. Lett. 2007, 98, 237201.

(29) He, Q. L.; et al. Tailoring exchange couplings in magnetic topological-insulator/antiferromagnet heterostructures. Nat. Mater. 2017, 16, 94-100.

(30) Wang, F.; Xiao, D.; Yuan, W.; Jiang, J.; Zhao, Y.-F.; Zhang, L.; Yao, Y.; Liu, W.; Zhang, Z.; Liu, C.; Shi, J.; Han, W.; Chan, M. H. W.; Samarth, N.; Chang, C.-Z. Observation of Interfacial Antiferromagnetic Coupling between Magnetic Topological Insulator and Antiferromagnetic Insulator. Nano Lett. 2019, 19, 2945-2952.

(31) Yin, L.; Wang, C.; Shen, Q.; Zhang, L. Strain-induced Curie temperature variation in $\mathrm{La}_{0.9} \mathrm{Sr}_{0.1} \mathrm{MnO}_{3}$ thin films. RSC Adv. 2016, 6, 96093-96102.

(32) Zhang, F.; Wu, B.; Zhou, G.; Quan, Z.-Y.; Xu, X.-H. Increased Curie temperature induced by orbital ordering in $\mathrm{La}_{0.67} \mathrm{Sr}_{0.33} \mathrm{MnO}_{3} /$ $\mathrm{BaTiO}_{3}$ superlattices. Nanoscale Res. Lett. 2018, 13, 24.

(33) Gupta, S.; Kanai, S.; Matsukura, F.; Ohno, H. Magnetic and transport properties of $\mathrm{Sb}_{2} \mathrm{Te}_{3}$ doped with high concentration of $\mathrm{Cr}$. Appl. Phys. Express 2017, 10, 103001.

(34) Wei, P.; Katmis, F.; Assaf, B. A.; Steinberg, H.; Jarillo-Herrero, P.; Heiman, D.; Moodera, J. S. Exchange-coupling-induced symmetry breaking in topological insulators. Phys. Rev. Lett. 2013, 110, 186807.

(35) Lang, M.; et al. Proximity induced high-temperature magnetic order in topological insulator-ferrimagnetic insulator heterostructure. Nano Lett. 2014, 14, 3459-3465.

(36) Alegria, L.; Ji, H.; Yao, N.; Clarke, J.; Cava, R. J.; Petta, J. R. Large anomalous Hall effect in ferromagnetic insulator-topological insulator heterostructures. Appl. Phys. Lett. 2014, 105, No. 053512.

(37) He, Q. L.; et al. Exchange-biasing topological charges by antiferromagnetism. Nat. Commun. 2018, 9, 2767.

(38) Wang, J.; Lian, B.; Qi, X.-L.; Zhang, S.-C. Quantized topological magnetoelectric effect of the zero-plateau quantum anomalous Hall state. Phys. Rev. B: Condens. Matter Mater. Phys. 2015, 92, No. 081107.

(39) Mogi, M.; Kawamura, M.; Yoshimi, R.; Tsukazaki, A.; Kozuka, Y.; Shirakawa, N.; Takahashi, K.; Kawasaki, M.; Tokura, Y. A magnetic heterostructure of topological insulators as a candidate for an axion insulator. Nat. Mater. 2017, 16, 516-521. 
(40) Deng, Y.; Yu, Y.; Shi, M. Z.; Guo, Z.; Xu, Z.; Wang, J.; Chen, X. H.; Zhang, Y. Quantum anomalous Hall effect in intrinsic magnetic topological insulator $\mathrm{MnBi}_{2} \mathrm{Te}_{4}$. Science 2020, 367, 895-900.

(41) Fu, H.; Liu, C.-X.; Yan, B. Exchange bias and quantum anomalous nomalous $\mathrm{Hall}$ effect in the $\mathrm{MnBi}_{2} \mathrm{Te}_{4} / \mathrm{CrI}_{3}$ heterostructure. Sci. Adv. 2020, 6, No. eaaz0948. 